\title{
An Approach for Utilizing Power Flow Modeling for Simulations of Hybrid Electric Propulsion Systems
}

\author{
Jeffryes W. Chapman* and Jonathan S. Litt ${ }^{\dagger}$ \\ NASA Glenn Research Center, Cleveland, OH, 44135, USA
}

This paper describes an approach to creating simulations of the electric components for a hybrid electric propulsion system. The proposed modeling technique is based on power/load flow modeling and is designed to provide a modular framework that includes buses, lines, and other electrical components that can be connected together to form the electrical distribution system. The purpose of this paper is to detail an electric distribution system modeling technique and to demonstrate how these models may be integrated with turbomachinery simulations. These general modeling techniques were created to be utilized for system and control design studies. Additionally, steady-state and dynamic performance for a proposed model example is compared with data from a hardware in the loop simulation.

Electrical current

Integral constant

Proportional constant

mile

Newton meter

Real power

Per unit

Reactive power

Radian

Revolutions per minute

Apparent power

Voltage

Admittance

Impedance

Voltage phase angle

Efficiency

Admittance angle

Torque

Rotational speed

\section{Nomenclature}

Side a or input

Side b or output

Error

Generator

Node number i

Node number $\mathrm{j}$

Load

\footnotetext{
* Aerospace Engineer, 3000 Aerospace Parkway, Brook Park OH, AIAA Member.

${ }^{\dagger}$ Research Engineer, 21000 Brookpark Rd., Cleveland OH, MS-77-1, AIAA Associate Fellow.
} 


\section{Introduction}

S planning for the next generation of aircraft engines continues, hybrid/turbo electric or fully electric propulsion systems are being explored by researchers with the aim of improving fuel efficiency, emissions, and noise levels. ${ }^{1}$ Examples of such engine concepts include the Boeing SUGAR Volt, ${ }^{2}$ ESAero ECO-150, ${ }^{3}$ NASA STARC-ABL,${ }^{4}$ and NASA N3-X. ${ }^{5}$ As newer concepts are developed, it is important to create adequate propulsion system level simulations that take into account the increasingly complex electrical system. This paper describes the development of a general use electrical distribution modeling framework that makes use of power flow or load flow techniques. This modeling framework was developed to be integrated with all other non-electric components of the propulsion system, in this case, turbomachinery (gas turbines and/or fans connected via shafts to electric motors and generators) will be considered. Many examples of electric system and component modeling may be found in literature ${ }^{6,7,8}$ This paper will describe the hybrid electric propulsion system modeling problem, highlighting simulation requirements and detailing a modeling technique that maintains a high degree of flexibility and an appropriate level of fidelity for system design or high level control design.

For this work, the electric or hybrid/turbo electric propulsion system is defined as a propulsion system where large sums of electrical power are moved through the system for the purpose of generating thrust or for other propulsion related reasons. For the systems considered, the main propulsors are fans that are run by gas turbine engines and/or electric motors connected in different configurations. In the case where a fan is run by an electric motor, the power may be taken from a battery or a generator attached to a gas turbine engine shaft. When the fan is run mainly by a gas turbine engine, electric energy may be added to or removed from the shaft/s to achieve desired performance at different operating conditions. Architectures for these systems can take many forms, therefore the modeling system needs to be flexible enough to accommodate any potential configuration. Generally, the major components considered for these systems are electric motors, electric generators, power electronics designed to convert power to a required medium, batteries, and gas turbine engine/s.

When creating a system level model of a hybrid electric propulsion system, the electric distribution system poses two main concerns: how efficient is the transport of power, and how do the dynamics of the electric system affect the non-electric components? To address the first concern, the model must contain a high fidelity power delivery system model. Many of these types of models have been created for generalized modeling needs and can be physics based, empirical, or a combination of the two. ${ }^{9}$ The second concern is a bit more nuanced because of the complexity of the dynamics within a propulsion system and the intended use for the simulation. In turbomachinery systems, dynamic performance may be affected by fast acting volume dynamics of the gas path, ${ }^{10}$ however, the most influential dynamic effect for system modeling is generally accepted, for most applications, to be the slower dynamics of the shaft. ${ }^{11}$ In the electrical portion of the system, many of the high frequency dynamics of the power electronics, generators, and motors are, similar to the turbomachinery, overshadowed by the relatively slow motor shaft dynamics. However, it is important to note that the two subsystems (electrical and turbomachinery) are also controlled based on component controllers (engine control, motor control, generator control, etc.) working together, therefore it is also plausible that interaction between the controller schemes creates shaft dynamics that need to be taken into account.

To address these electric subsystem modeling requirements, the power/load flow technique was utilized. Power flow is a steady-state method of modeling electrical systems. These models are based around lines linking different buses that are connected to generators or loads. ${ }^{6}$ Power flow modeling provides a number of advantages: the overall system is modular, allowing for components to be added or removed easily, interaction with the model may come in the form of a specified power and/or current and voltage, components may be represented by equivalent circuits that match required fidelity, and simulation execution time is fairly fast. Although these types of models come with many attractive attributes, they also assume the model is in steady state. In this paper, the limitation and applicability of the power flow modeling framework is compared to test data taken from the NASA Electric Aircraft Test bed (NEAT) ${ }^{12}$ facility, and introduction of dynamics that influence the applied shaft torque are explored. This combination of modeling techniques results in a quasi-steady state modeling format that could be used for steady-state performance prediction, and dynamic system responses for system studies or control system design. It should be noted that this modeling technique is not developed for electrical subsystem control design, as this would require that a fully dynamic simulation of the electrical subsystem be created.

Implementation of this electrical modeling scheme was completed within MATLAB ${ }^{\circledR} /$ Simulink $^{\circledR}$ with the assumption that that the subsystem models would be integrated with an engine model created with the Toolbox for the Modeling and Analysis of Thermodynamic Systems (T-MATS). An add-on to Simulink, T-MATS is an open 
source software that facilitates the creation of thermodynamic systems that require local solvers. ${ }^{13, \ddagger}$ This modeling approach utilizes an energy balance method and component performance tables or maps to define the non-linear turbomachinery system. In addition to gas turbine modeling capability, T-MATS offers a flexible local solver that will be used to solve the power flow problem.

Subsequent sections of this paper detail the development of the power flow modeling equations, and several model examples. A simple comparison between electric system data gathered from NEAT will also be shown. Specifically, discussion of the electric modeling technique is located in Section II, followed by a description of a method to integrate the electric system with the mechanical system in Section III. An analysis of the steady state and dynamic operation using test data appears in Section IV. Finally, the conclusions are given in Section V.

\section{Adjusted Power Flow Modeling}

Power/load flow modeling is well documented, however the highly coupled nature of the problem makes solving these problems in MATLAB/Simulink a challenge due to the directional flow nature of Simulink. This section describes the classic power flow problem and then describes how the techniques were adjusted to work within Simulink.

Power flow modeling makes use of node and line building blocks that may be connected together to form complex systems. Each node signifies a connection point and each line describes the connection of two nodes. Nodes are defined by a single voltage value and a power value that is dissipated or generated at that node via some external system source. Power at the nodes is defined by the generalized power flow equation shown in Eq. (1), derived in Ref. [6].

$$
\begin{aligned}
& P_{G i}=P_{L i}+\sum_{j=1}^{n} V_{i} V_{j} Y_{i j} \cos \left(\delta_{i}-\delta_{j}-\gamma_{i j}\right) \\
& Q_{G i}=Q_{L i}+\sum_{j=1}^{n} V_{i} V_{j} Y_{i j} \sin \left(\delta_{i}-\delta_{j}-\gamma_{i j}\right)
\end{aligned}
$$

With variables defined as: $P_{G i}$ is node generated real power, $P_{L i}$ is node load real power, $Q_{G i}$ is node generated apparent power, $Q_{L i}$ is node load apparent power, $i$ is the current node number, $j$ is a connecting node number, $V_{i}$ is voltage at node $i, V_{j}$ is voltage at connected node $j, Y_{i j}$ are the entries to the admittance matrix ([Y]), which contains all the line circuit information (see Ref. [6] for more details), $\delta_{i}$ is the phase angle at node $i, \delta_{j}$ is the phase angle at connected node $j$, and $\gamma_{i j}$ is the phase angle of the line connecting nodes $i$ and $j$. In solving this problem, connection admittances and load power losses may be assigned assumed values, leaving generated powers and voltages to be identified. To aid in defining these variables, three main types of nodes are defined: slack, load, and generator. The slack node is similar to a generator where the voltage is specified and the powers are assumed to be provided (the generator "picks up the slack" in the system). At a load node, a power loss is defined, generated powers are assumed zero, and a voltage value for the node is calculated. The generator node uses an assumed generated power and voltage to determine the imaginary components of each. Once the system has been determined, numerical methods must be used to solve for the unknown values at each node.

As mentioned before, this formulation of power flow creates a highly coupled modeling scheme. To circumvent this complication and create a modeling scheme that works with a modular directional flow modeling technique (such as MATLAB/Simulink), the derivation above needs to be adjusted. In creating this scheme, one must realize that the nodal power defined above is simply the product of the nodal voltage and the summation of all currents entering the node. This realization allows a modeling formulation in which each line component obtains the upstream node voltage then determines the downstream node voltage to calculate the current, effectively removing the need for full system definition and allowing for modular components. For the model definition to work, a local solver needs to be introduced to determine the downstream voltages. For this work, a Newton Raphson type solver (provided by T-MATS) was utilized. This localized solver determines a set of independent variables by monitoring and forcing dependent variables to be equal to zero. In this case, it is necessary that the number of independents match the number of dependents. A list of the generalized power flow components and their typical independents and dependents is provided in Table 1. Definitions of their complete component equations are detailed in the following paragraphs.

\footnotetext{
${ }^{\ddagger}$ https://github.com/nasa/T-MATS/releases , cited 12/2017
} 
Table 1. Generalized AC power flow components

\begin{tabular}{|l|c|c|}
\hline \multicolumn{1}{|c|}{ Component } & Independents & Dependents \\
\hline Impedance, line & $V_{b}, \delta_{b}$ & none \\
\hline Transmission line, line & $V_{b}, \delta_{b}$ & none \\
\hline Transformer, line & $V_{b}, \delta_{b}$ & none \\
\hline Slack bus, node & none & none \\
\hline Load bus, node & none & $P_{e r}, Q_{e r}$ \\
\hline Generator bus, node & none & $P_{e r}, V_{e r}$ or $Q_{e r}$, see below \\
\hline
\end{tabular}

The impedance component offers the basis of any line by allowing any impedance to be defined manually. The component is created using an upstream voltage input, then solving for the downstream voltage. The basic equation that governs the block is shown in Eq. (2). Note: $\bar{V}_{b}$ is an independent value and is therefore determined by the local solver, $\bar{I}_{a}$ is complex (side a) current for a line, $\bar{I}_{b}$ is complex output (side b) current for a line, $\bar{V}_{a}$ is complex (side a) voltage, $\bar{V}_{b}$ is complex output (side b) voltage, and $\bar{Y}$ is complex admittance (or the inverse of impedance, Z) .

$$
\begin{aligned}
& \bar{I}_{b}=\bar{Y} *\left(\bar{V}_{b}-\bar{V}_{a}\right) \\
& \bar{I}_{a}=\bar{Y} *\left(\bar{V}_{a}-\bar{V}_{b}\right)
\end{aligned}
$$

For especially long transmission lines ( $>50 \mathrm{mi}$.) the impedance component can be updated to include a small amount of admittance (typically reactive) added to each port and run to ground. The following equation, Eq. (3), shows how this is formulated. This formulation is developed mostly for the example problem as most propulsion systems will not have transmission lines that are longer than 50mi.

$$
\begin{aligned}
& \bar{I}_{b}=\left(\bar{Y}_{\text {line }}+\bar{Y}_{\text {ground }}\right) * \bar{V}_{b}-\bar{Y}_{\text {line }} * \bar{V}_{a} \\
& \bar{I}_{a}=\left(\bar{Y}_{\text {line }}+\bar{Y}_{\text {ground }}\right) * \bar{V}_{a}-\bar{Y}_{\text {line }} * \bar{V}_{b}
\end{aligned}
$$

The transformer component allows the conversion between one voltage level and another. The component considers a two-winding transformer utilizing a pi equivalent circuit taken from Ref. [6]. The basic equation that governs the block is shown in Eq. (4). Note: c is per-unit turns ratio.

$$
\begin{gathered}
\bar{I}_{b}=-\bar{c}^{*} * \bar{Y} * \bar{V}_{a}+c^{2} * \bar{Y} * \bar{V}_{b} \\
\bar{I}_{a}=\bar{Y} * \bar{V}_{a}+\bar{c} * \bar{Y} * \bar{V}_{b}
\end{gathered}
$$

The slack bus component offers a general purpose starting point to the model that will account for any power disparities in the system. This block acts as a way of specifying the voltage of the system, and uses the summation of all input and output currents to determine a complex power. The basic equation that governs the block is shown in Eq. (5).

$$
\begin{gathered}
\bar{V}_{b}=\bar{V}_{a} \\
\bar{S}=\bar{V}_{a} *\left(\sum \bar{I}\right)^{*}
\end{gathered}
$$

The load bus component enables the addition of external known power loads. For this block, a complex power load is defined. This complex power is then compared with a calculated complex power input to determine a power error that can be used to back calculate the system voltages. The basic equations that govern the block are shown in Eq. (6). Note: $P$ and $Q$ are calculated values, $P_{\text {load }}$ and $Q_{\text {load }}$ are known values, and $P_{e r}$ and $Q_{e r}$ are dependents to be solved for by the solver.

$$
\begin{gathered}
\bar{V}_{b}=\bar{V}_{a} \\
\bar{S}=\bar{V}_{a} *\left(\sum^{*} \bar{I}\right)^{*}=P+j Q \\
P_{e r}=P-P_{\text {load }} \\
Q_{\text {er }}=Q-Q_{\text {load }}
\end{gathered}
$$

The generator bus component enables the addition of external known real power generation at a regulated voltage. For this block, a real generated power at a specified voltage is defined. This real power is then compared with a calculated real power to determine a power error, and input voltage is compared with the specified voltage to determine phase angle and reactive power. The basic equations that govern the block are shown in Eq. (7). Note: $P_{\text {load }}$ and $V_{\text {specified }}$ are known values, $P$ and $Q$ are independents solved for by the solver, and $P_{e r}$ and $V_{e r}$ are dependents to be forced to zero by the solver. As an additional complexity, there also exists a maximum reactive power that once met becomes a limit. For more information on this limit see Ref. [6]. 


$$
\begin{gathered}
\bar{V}_{b}=\bar{V}_{a}=V \angle \delta^{\circ} \\
\bar{S}=\bar{V}_{a} *\left(\sum \bar{I}\right)^{*}=P+j Q \\
P_{e r}=P-P_{\text {load }} \\
V_{\text {er }}=V-V_{\text {specified }}
\end{gathered}
$$

To demonstrate the above components, a 5 bus system was created based on literature. ${ }^{14} \mathrm{~A}$ simple block diagram of this system is shown in Figure 1 and implemented in Figure 2 with node voltages and phase angles summarized in Table 2. These figures and tables validate the adjusted modular power flow modeling scheme and show that this method may be used for the classic power flow problem.

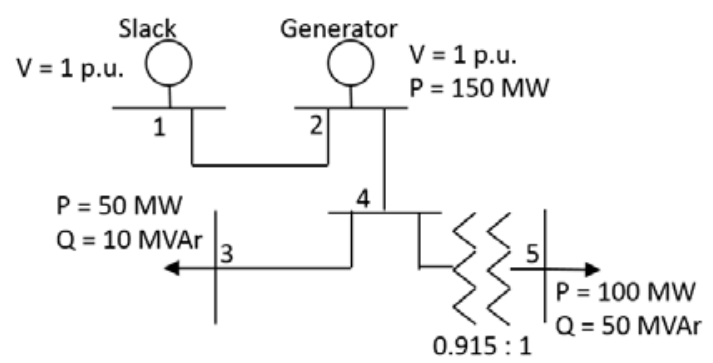

Table 2. Generalized AC power flow components

All lines: $\mathrm{Z}=0.001+\mathrm{j} 0.05$ with $\mathrm{Z} 2=\mathrm{j} 20$

\begin{tabular}{|c|c|c|}
\hline Node-Type & V(p.u.) & $\boldsymbol{\delta}$ (rad) \\
\hline 1 - slack & 1 & 0 \\
\hline 2 - Generator & 1 & -0.0001541 \\
\hline 3 - Load & 0.9607 & -0.1043 \\
\hline 4 - Load & 0.9644 & -0.07739 \\
\hline 5 - Load & 0.9991 & -0.1725 \\
\hline
\end{tabular}

Figure 1. Example power flow block diagram.

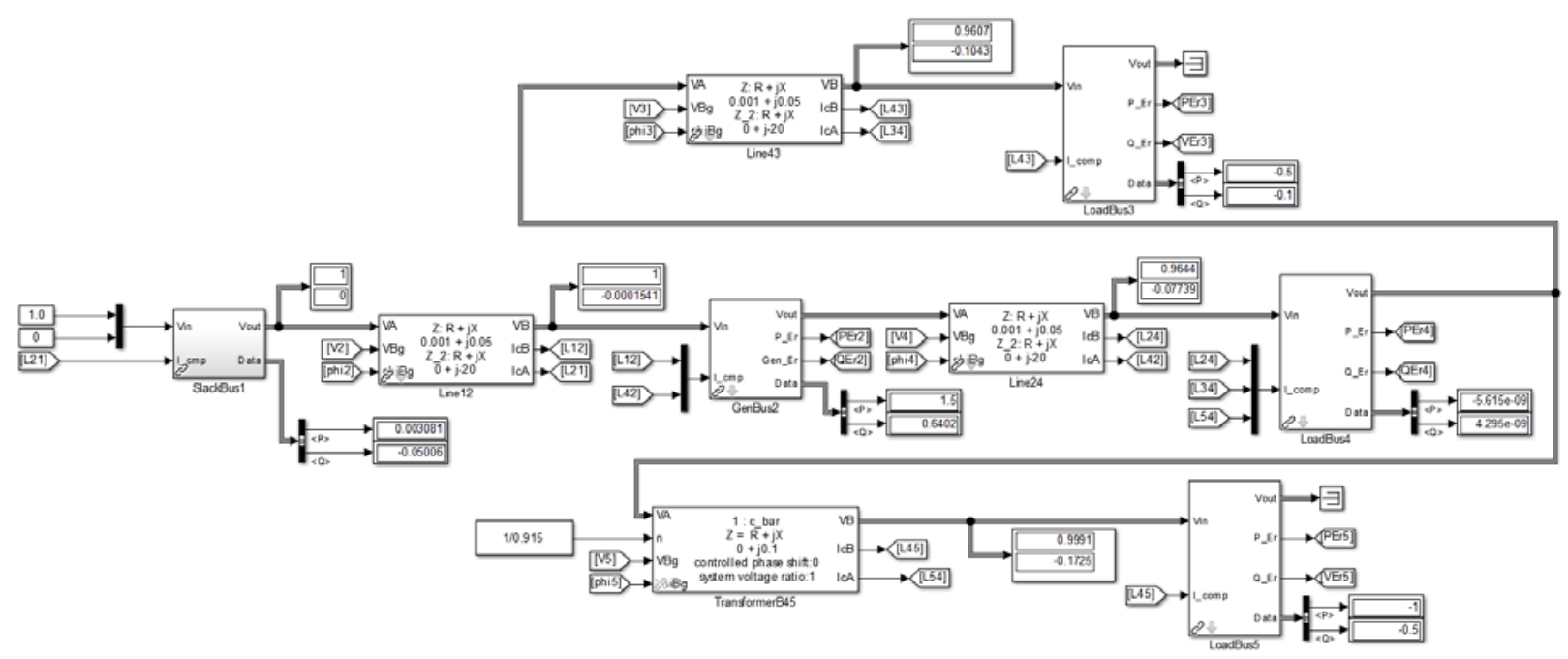

Figure 2. Example power flow simulation implemented in MATLAB/Simulink. 


\section{Integration of Power Flow with Turbomachinery Models}

The integration of the power flow model with the non-electric components of a hybrid electric or electric machine is performed by adjusting the load and/or generator buses within the electrical distribution model to account for the mechanical subsystems. For example, in the case of a gas turbine, additional power could be taken from the shaft in the form of torque and sent to the electrical system generator, leaving the turbine to account for any torque debit. Similarly, power loss from a load bus may be used to run a fan, or power gain from a generator node may be used to represent a battery. In these systems, a slack node could be used to stabilize the main system bus, while known output generation devices could be considered as a generator bus. Often these systems contain a combination of AC and DC components. This complication is rectified by removing the reactive components of the equations and introducing AC to DC converters as needed. ${ }^{15,16}$

As a starting point for these power transfer systems, three new components were considered: a battery, a motor and a generator. In the simplest terms, the battery may be considered simply a DC generator node or DC slack node, therefore no additional modeling was required. The motor and generator were assumed to operate similarly and relate electrical power to mechanical torque by the relationship shown in Eq. (8), where $P$ is power, $\eta$ is efficiency, $\tau$ is torque, and $\omega$ is rotational speed.

$$
P=\eta \tau \omega
$$

In this simplified modeling, the power electronics that convert to the required electric voltage and frequency for a given shaft speed and torque requirement are assumed to be accounted for by the component efficiency. This efficiency

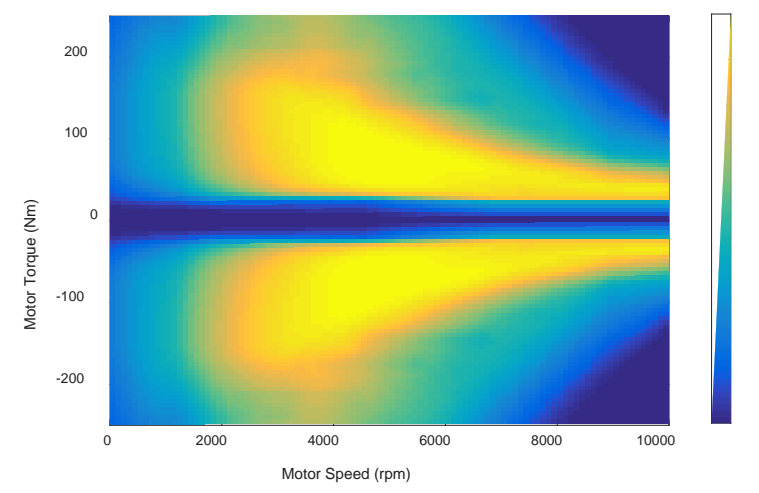

Figure 3. Efficiency map of induction motor system. ${ }^{9}$

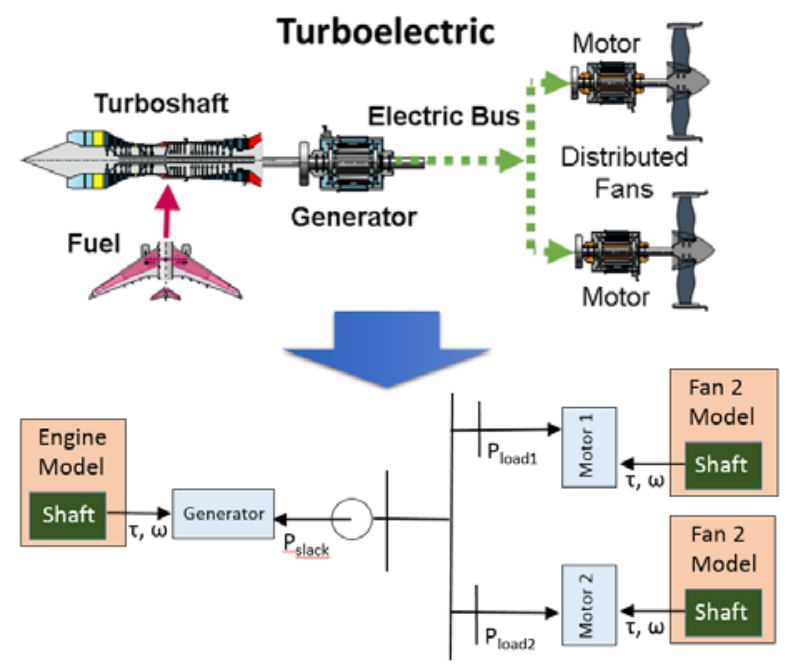

Figure 4. Example architecture of a turbo electric propulsion system. ${ }^{1}$ is calculated empirically based on the efficiency map of the motor or generator. An example of an induction motor/generator efficiency map is shown in Figure 3. In this figure, positive torque denotes a motor acting in propulsion mode or a motor that is driving a load, and the negative torque denotes a motor acting in regenerative mode or acting as a generator. Generally it can be seen that low torques and speeds have low efficiency, medium speeds with medium and high amounts of torque have high efficiency, and high speeds maintain high efficiency only in a narrow band of relatively low torque. Although, this map shows a symmetrical efficiency about zero torque, this is not necessarily the case for all motors. This derivation of the electric motor model is a steady-state approach. Internal dynamics due to high frequency switching and inner loop and outer loop control tuning are neglected with the assumption that their effects are small or are damped out by the shaft dynamics. This assumption will be tested in the following sections.

As an example of this integration strategy, Figure 4 shows a high level subsystem diagram highlighting the links between models for an example turbo electric propulsion system taken from Ref [1]. In this system architecture, a turboshaft is connected to a generator that then supplies the power to two separate fans. To model this, four distinct models are created: an engine model, an electric distribution model, and two fan models. The interfaces between the different subsystems are handled by motor and generator models that translate shaft torque and speed into power. This modular approach also allows each model to be developed and updated separately to a fidelity that is appropriate for the given application. 


\section{System Dynamics and Model Matching}

The electric components in hybrid electric propulsion systems have many sources of dynamics that can be taken into account. The most common examples of these dynamics are voltage/current for AC electrical flows, power electronics switching for methods such as pulse width modulation, motor/generator torque and speed controller performance, and motor/generator shaft dynamics. In an effort to simplify the modeling requirements it is desirable to take into consideration only the dynamics necessary when creating a quasi-steady state simulation. Determining what model dynamics are necessary requires an understanding of model's purpose and of which component dynamics dominate the system response. For turbomachinery system models, the dominant dynamics are generally considered to be shaft dynamics because of their dampening effect on faster system transients and their connection to critical propulsion system properties, such as output thrust and turbomachinery safety criteria, e.g., stall margin or critical temperatures. This paper will take a similar approach in determining what dynamics are appropriate for the electrical portions of the propulsion system, i.e., only dynamics that are shown to affect the system's shaft speeds will be considered.

To understand the responses of an electro-mechanical system, it is important to have an understanding of the electric motor. The electric motor geometry is considered to be a rotating shaft surrounded by a sheath considered a stator. Motor torque is generated by the interaction between currents and magnetic fields within the shaft and stator. These currents and magnetic fields are generated by driving AC current through sets of windings located around the shaft and/or stator, or with the use of a permanent magnet. An image of a synchronous motor with 6 sets of windings and a 2 pole permanent magnet rotor is shown in Figure 5. Motor speed and torque control is managed by adjusting the magnitude and frequency of the AC voltage across each winding. A notional diagram of a synchronous motor control system is shown in Figure 6. In this control system, a shaft speed is being requested by an outside source. This requested speed is compared to the current speed, and a speed controller generates a torque request. Similarly, the torque request is compared with a torque calculated from the measured winding currents to generate a current request. The current request is then compared to the actual winding currents to determine a voltage demand. This voltage demand is sent to an inverter that converts a DC voltage maintained by the power supply to the required AC voltage, magnitude and frequency. Dynamic responses for the system include shaft dynamics from the motor, switching dynamics from the inverter, and controller dynamics from the three control loops.

The interaction between the different sources of dynamics was explored by comparing data generated from a quasisteady state model that takes into account only shaft dynamics and simplified motor controls, with electric motor data gathered from the NEAT facility. The NEAT facility is being designed to facilitate full-scale electric aircraft powertrain development and testing, and contains a wide range of electric propulsion hardware.

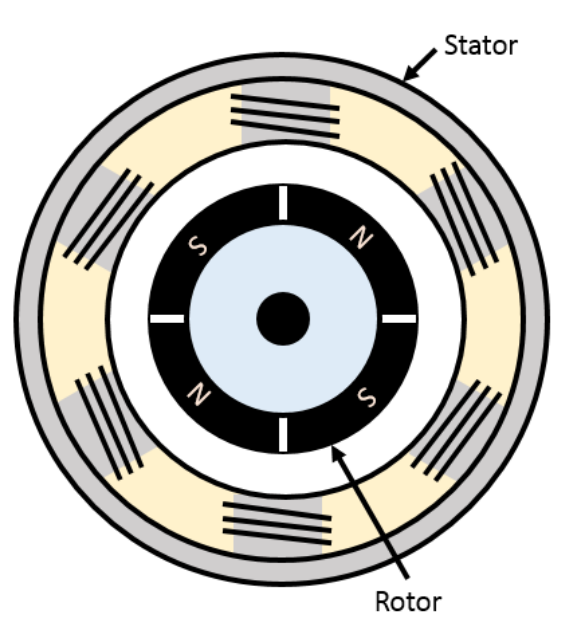

Figure 5. Stator and rotor diagram of a synchronous motor system. Configuration shows a rotor with 2 pairs of poles and a stator with 6 coils.

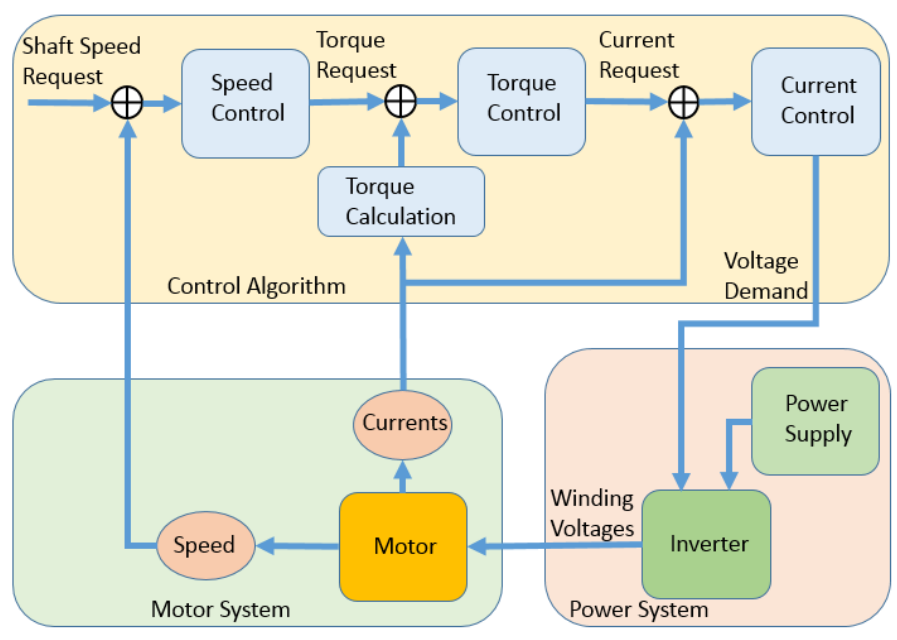

Figure 6. Notional synchronous motor control system. 


\section{Torque}

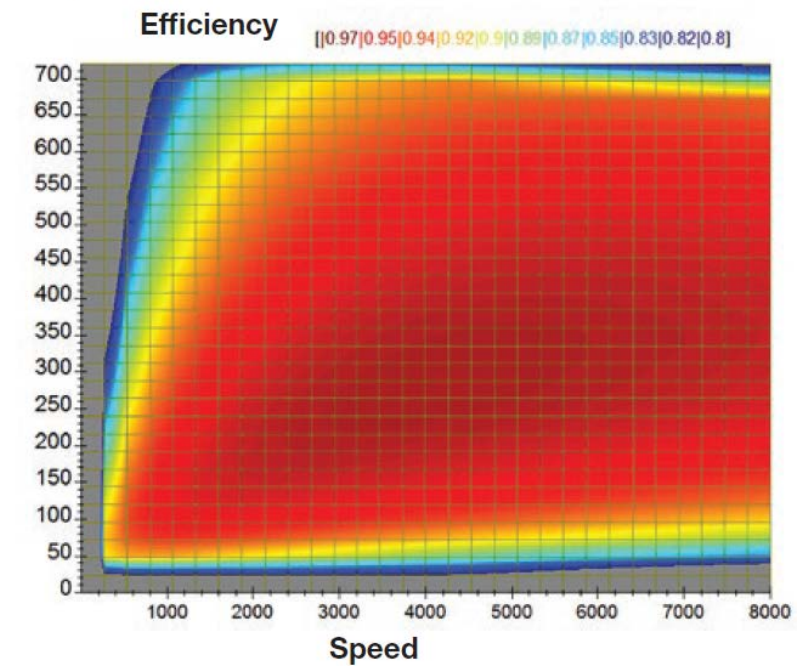

Figure 7. GVM210 motor efficiency map. ${ }^{17}$
For this study, a two motor architecture was utilized. In this architecture the two motors are connected via a shaft then connected to a bus that is run to a power source. One motor is controlled to a specified shaft speed while the other is controlled to a specified torque. During operation, the speed side motor draws power from the bus to push the shaft, and the torque side motor draws power from the rotating shaft and returns it to the bus. Starting power and power lost due to efficiency losses are taken from the power supply. A schematic of the system is shown in the appendix. The synchronous motors used for this test were made by Parker Hannifin (Part Number GVM210-300Q6) ${ }^{17}$ and are driven by motor controllers made by Rinehart (Model PM100). The motor controller efficiency is listed within the specification as $97 \%$, and the motor efficiency map is shown in Figure 7. This efficiency map demonstrates a wide range of torque and speed values with efficiencies between $97 \%$ and $80 \%$.

A diagram of the representative simulation is shown in Figure 8. This simulation considers a DC system with inverters contained with the motor or generator models as described by the power relation above, Eq. (8). The setup for the model consists of a load bus interfacing with the speed and torque controlled motors, and a slack bus used to represent the power supply. Buses are electrically connected to each other via resistive lines. Torque from the two motors is used to determine shaft acceleration, which is then integrated to update the shaft speed. The two motor controllers (torque and speed) are approximated with proportional integral (PI) controllers utilizing feedback from the motor models and issuing a power demand to each motor. Tunings developed for these PI controllers were $100 / 400$ and $100 / 1000 \mathrm{~K}_{\mathrm{p}} / \mathrm{K}_{\mathrm{i}}$, for speed and torque motors respectively. Power supply voltage is set to a constant value based on the line specification for the test.

A steady state comparison of NEAT facility data and the model was performed on voltages, currents, torques, and speeds. In this test, the DC bus voltage was set to $600 \mathrm{~V}$, speed demands were adjusted to 1000, 3000, and $5000 \mathrm{rpm}$ at torques of 100, 200, 250 (for $5000 \mathrm{rpm}$ only), and $260 \mathrm{Nm}$ (for $1000 \mathrm{rpm}$ and $3000 \mathrm{rpm})$. Simulation line resistances were set to 0.015 and 0.035 Ohms for the speed side and torque side lines respectively. Results of the comparison are shown in Figure 9. Differences in shaft speeds between the two models is negligible, which is not surprising because the control systems are controlling the speed and torque values. Simulated speed side voltages have steady state values that are slightly lower than the NEAT data, but all points follow a similar trend to the model results. Torque side differences are larger than speed side differences with some points that don't follow the model trend. These voltage differences were deemed acceptable because of the small total changes in voltage, less than $1 \%$, and noise content of the voltage sensors. Matching between current data sets was generally very good. Torque side current values were nearly identical for

Figure 8. Test case simulation architecture. 


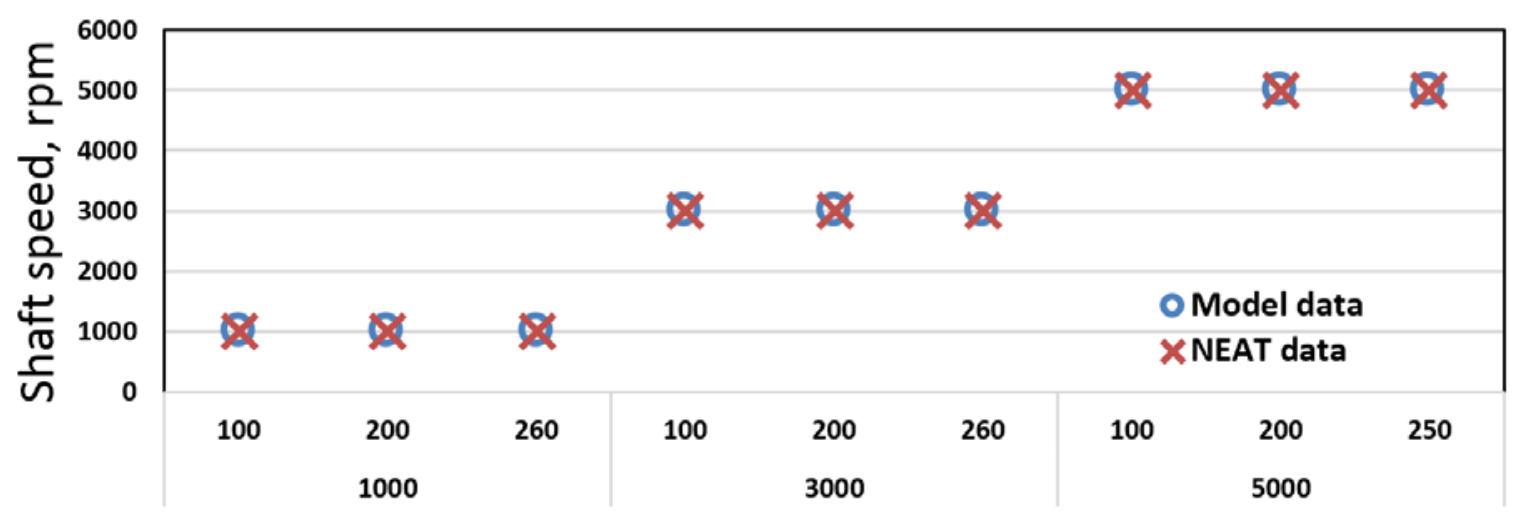

Torque demand, $\mathrm{Nm}$

Shaft speed demand, rpm
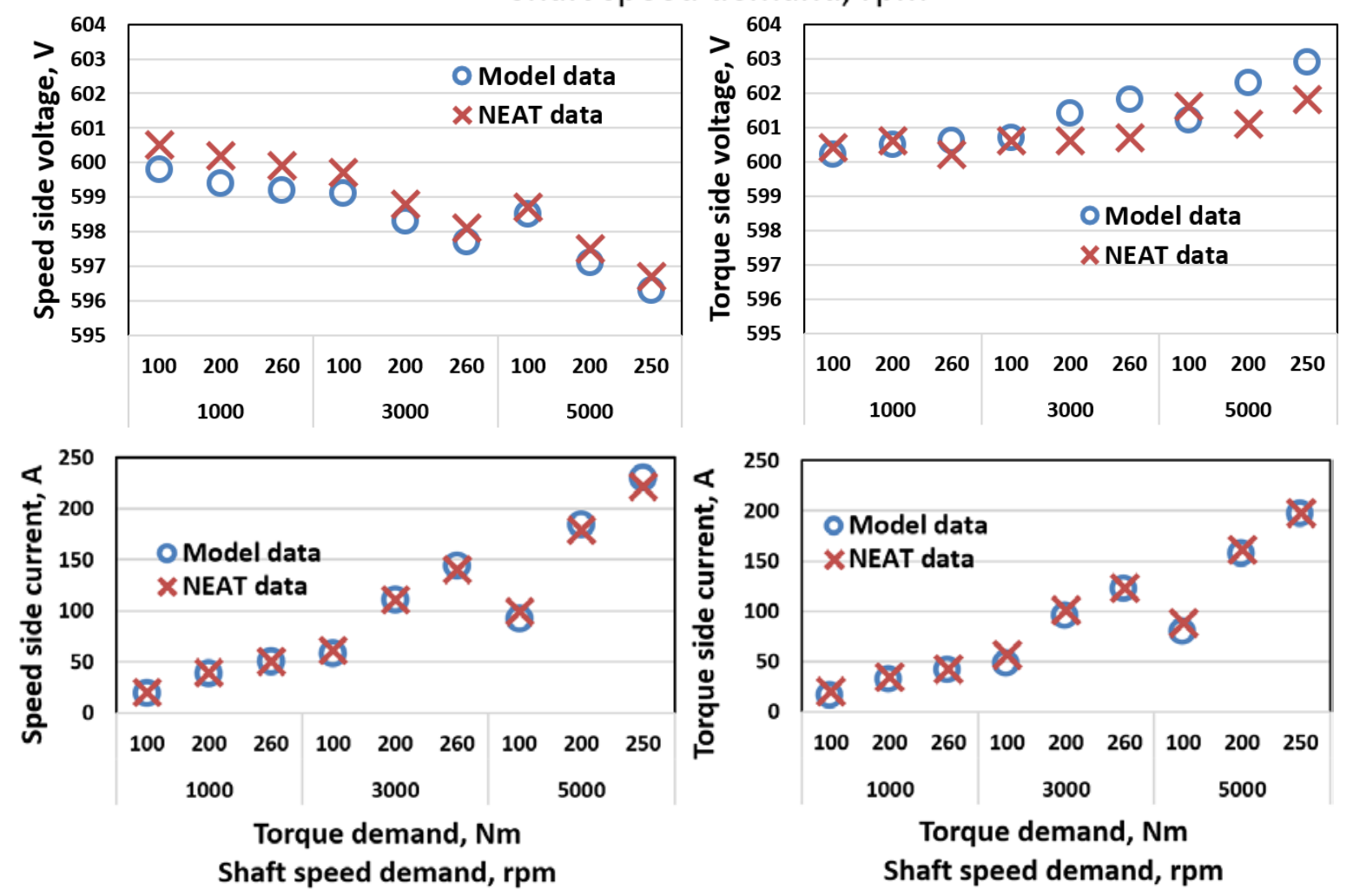

Figure 9. Steady state comparison of model vs. NEAT facility data.

most points, however current at higher power levels on the speed side motor are larger, with the maximum difference at about $10 \mathrm{~A}$.

Simulation and facility data were also compared for a burst torque transient. In this test, the shaft was driven to a speed of $1000 \mathrm{rpm}$, while the torque side motor generated $100 \mathrm{Nm}$ of torque. This torque was then increased at $1 \mathrm{~s}$ to a value of $150 \mathrm{Nm}$, while the desired speed was held constant at $1000 \mathrm{rpm}$. Traces of the performance can be seen in Figure 10. Looking at the speed side shaft torque, overshoot may be observed within both transients. Peak torque predicted by the model is roughly $20 \mathrm{Nm}$ higher than the facility data, however this is very short and the facility sampling time may have reduced the observed peak. Both model and facility shaft speed transients show a reductions of about $80 \mathrm{rpm}$ and recovery within $0.75 \mathrm{~s}$ with no overshoot. Model bus voltage was essentially constant because no dynamics were placed into the model to account for them. A facility bus voltage transient may be observed where the voltage increases $5 \mathrm{~V}$, then drops below $590 \mathrm{~V}$ before recovering. Electrical current traces show overshoot immediately 

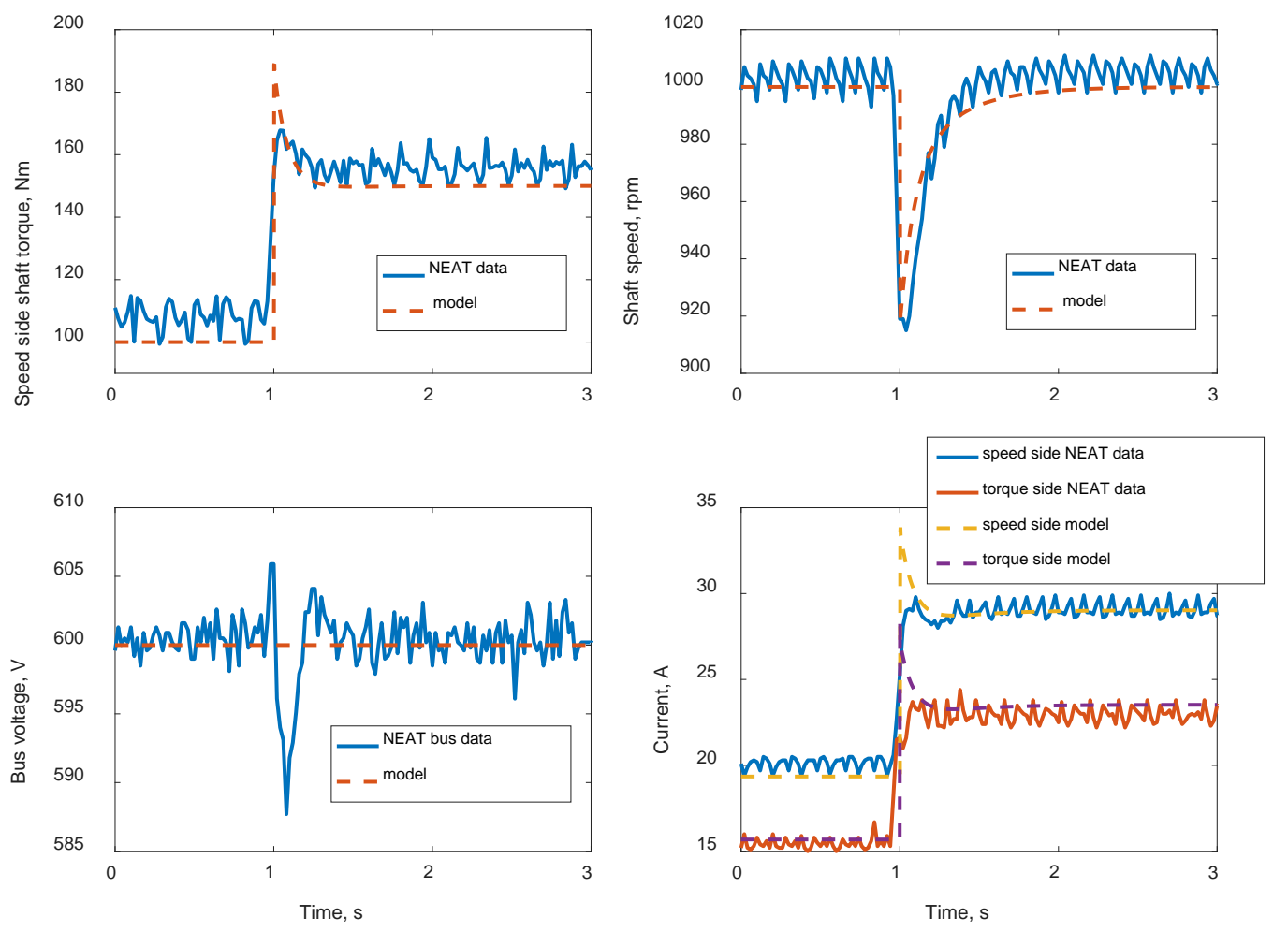

Figure 10. Comparison of model vs. NEAT facility data for a 100 to $150 \mathrm{Nm}$ torque step change at $1000 \mathrm{rpm}$.

after the torque demand is increased. The overshoot in model current is on the order of $15 \%$, which may be attributed to the lack of a voltage transient. These traces generally show a model that tracks shaft speed and torque fairly accurately, however much of the electrical transient effects are not represented. As stated previously, this modeling effort was only concerned with mechanical shaft speed and torque, so these results were deemed acceptable. If more accurate transients are required within the electrical system, dynamics could be added to the power supply system to account for the bus voltage fluctuations. It should be noted that the portions of the transients unaccounted for within the torque, current, and voltage occur very quickly relative to the shaft dynamics, therefore it is unlikely these transients will have an effect on the turbomachinery performance unless they cause electric system to hit limits that have larger system implications, such as for electric system sizing studies.

\section{Conclusions}

This paper details development of a method of electric system modeling for hybrid, turbo, or fully electric propulsion system simulations. With this modeling technique, an adjusted power/load flow method is used to simulate the electric distribution system and electro-mechanical component models, such as electric motors and generators, to enable integration with mechanical sub-systems. This power flow technique is updated to operate within the MATLAB/Simulink environment utilizing local solvers from the open source Toolbox for the Modeling and Analysis of Thermodynamic Systems (T-MATS), and is shown to be capable of solving the power flow problem. Equations that model the behavior of electro-mechanical devices such as motors and generators are also described and used to integrate these models with the full system. To demonstrate the power flow modeling technique, a simple five bus electric distribution system is solved. Additionally, a quasi-steady state model of a linked two motor test from the NASA Electric Aircraft Test bed (NEAT) was created to show how steady-state and appropriate transient responses of system hardware may be represented by the simulation strategy. This simulation demonstrates that turbomachinery shaft speed responses may be simulated taking into account only shaft dynamics and utilizing simplified motor control delay, however some electrical system transients will be neglected. This type of model could work well for system level or control system studies where the electrical system limits are not an issue and simulation run time is a concern. 


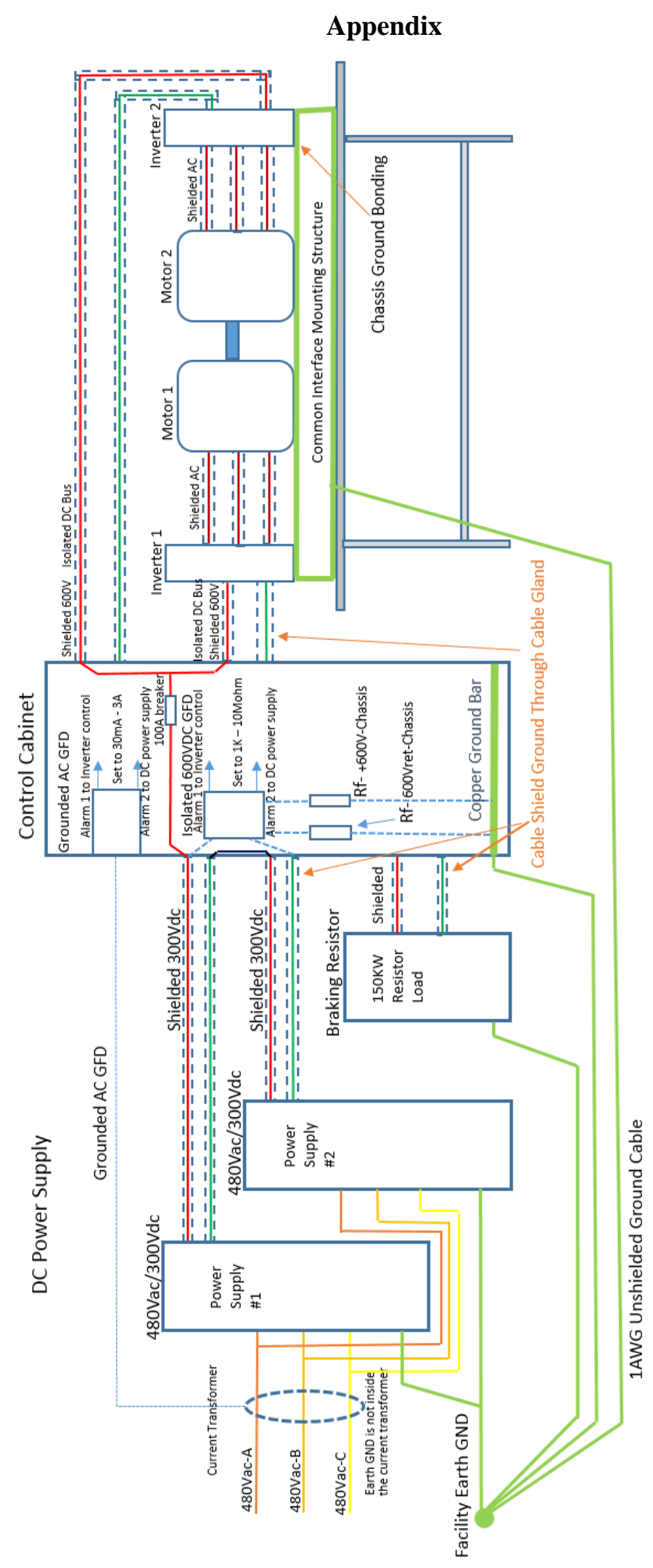

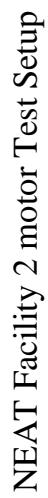




\section{Acknowledgments}

The authors would like to thank the NASA Transformational Tools and Technologies (TTT) project, under the Transformative Aeronautics Concept Program (TACP) for the funding of this work. Additionally, the authors would like to thank Ying Cha and Scott Lawyer of Vantage Partners, and Rodger Dyson of NASA Glenn Research Center for their contribution of the NEAT facility system schematic located in the appendix.

\section{References}

${ }^{1}$ Jansen, R.H., Bowman, C., Jankovsky, A., Dyson, R., Felder, J., “Overview of NASA Electrified Aircraft Propulsion Research for Large Subsonic Transports,” 53rd AIAA/SAE/ASEE Joint Propulsion Conference, AIAA Propulsion and Energy Forum, Atlanta, AIAA-2017-4701, GA, June 25-29, 2017.

${ }^{2}$ Bradley, M. K., and Droney, C. K., "Subsonic Ultra Green Aircraft Research: Phase II—Volume II—Hybrid Electric Exploration,” NASA/CR-2015-218704, 2015.

${ }^{3}$ Schiltgen, B. T., Freeman, J. L., and Hall, D. W., “Aeropropulsive Interaction and Thermal System Integration within the ECO150: A Turboelectric Distributed Propulsion Airliner with Conventional Electric Machines,” AIAA Aviation Technology, Integration and Operations Conference, AIAA 2016-4064, AIAA, Reston, VA, 2016.

${ }^{4}$ Welstead, J., and Felder, J. L., "Conceptual Design of a Single-Aisle Turboelectric Commercial Transport with Fuselage Boundary Layer Ingestion,” 54th AIAA Aerospace Sciences Meeting, AIAA, Reston, VA, 2016.

${ }^{5}$ Felder, J. L., Brown, G. V., and Kim, H. D., "Turboelectric Distributed Propulsion in a Hybrid Wing Body Aircraft," 20th International Society for Airbreathing Engines, ISABE-2011-1340, Gothenburg; Sweden, 2011.

${ }^{6}$ Gross, C.A., Power System Analysis, John Wiley \& Sons, New York, NY, 1998.

${ }^{7}$ Ong, C.M., Dynamic Simulation of Electric Machinery Using MATLAB/Simulink, Prentice Hall, Upper Saddle River, NJ, 1998.

${ }^{8}$ Perullo, C.A., Trawick, D., Clifton, W., Tai, J.C.M., Mavris, D.N.,’Development of a Suite of Hybrid Electric Propulsion Modeling Elements Using NPSS,” GT2014-27047, Proceedings of ASME Turbo Expo, Dusseldorf, Germany, Jun. 16-20, 2014.

${ }^{9}$ Liu, W., Introduction to Hybrid Vehicle System Modeling and Control, John Wiley \& Sons, Hoboken, NY, 2013.

${ }^{10}$ Hale, A. A; Davis, M. W. Jr; “Dynamic Turbine Engine Compressor Code, DYNTECC - Theory and Capabilities.” AIAA923190, AIAA Joint propulsion Conference, Nashville, TN, July 1992.

${ }^{11}$ Jaw, Link C., with Jack Mattingly, Aircraft Engine Controls: Design, System Analysis, and Health Monitoring, American Institute of Aeronautics and Astronautics, Inc, VA, 2009.

${ }^{12}$ Dyson, R.W., “NASA Electric Aircraft Test bed (NEAT) Development Plan-Design, Fabrication, Installation,” NASA/TM-2016219085, July, 2016.

${ }^{13}$ Chapman, J.W., Lavelle, T.M., May, R.D., Litt, J.S., Guo, T.H., “Toolbox for the Modeling and Analysis of Thermodynamic Systems (T-MATS) User’s Guide,” NASA/TM-2014-216638, 2014.

${ }^{14}$ Expósito, G., Conejo, A., Cañizares, C., Electric Energy Systems. Analysis and Operation, CRC Press, Boca Raton, Florida, 2008.

${ }^{15}$ Ahmed, H., Eltantawy, A., Salama, M., "A Generalized Approach to the Load Flow Analysis of AC-DC Hybrid Distribution Systems,” IEEE Transactions on Power Systems, Volume: PP, Issue: 99, June 28, 2017.

${ }^{16}$ Smed, T., Anderson, G., Sheblé, G.B., Grigsby, L.L., “A New Approach to AC/DC Power Flow,” IEEE Transactions on Power Systems, Volume: 6, Issue: 3, August, 1991.

${ }^{17}$ Parker Hannifin, Global Vehicle Motor - GVM Specification, 192-300108N6, 688358. 